\title{
Modeling and Analysis of Security Trade-Offs - A Goal Oriented Approach
}

\author{
Golnaz Elahi ${ }^{1}$, Eric $\mathrm{Yu}^{2}$ \\ ${ }^{1}$ Department of Computer Science, University of Toronto, Canada, M5S 1A4 \\ gelahi@cs.toronto.edu \\ ${ }^{2}$ Faculty of Information, University of Toronto, Canada, M5S 3G6 \\ eric.yu@utoronto.ca
}

\begin{abstract}
In designing software systems, security is typically only one design objective among many. It may compete with other objectives such as functionality, usability, and performance. Too often, security mechanisms such as firewalls, access control, or encryption are adopted without explicit recognition of competing design objectives and their origins in stakeholders' interests. Recently, there is increasing acknowledgement that security is ultimately about trade-offs. One can only aim for "good enough" security, given the competing demands from many parties. This paper investigates the criteria for a conceptual modeling technique for making security trade-offs. We examine how conceptual modeling can provide explicit and systematic support for modeling and analyzing security trade-offs. We examine several existing approaches for dealing with trade-offs and security trade-offs in particular. From analyzing the limitations of existing methods, we propose an extension to the i* Framework for security trade-off analysis, taking advantage of its multiagent and goal orientation. The method was applied to several case studies used to exemplify existing approaches. The resulting models developed using different approaches are compared.
\end{abstract}

Keywords: Security Trade-offs, Trade-off Analysis, Conceptual Modeling, Goal Modeling, Goal Model Evaluation.

\section{Introduction}

"Security is about trade-offs, not absolutes."

Ravi Sandhu

In designing software systems, security is typically only one design objective among many. Security safeguards may conflict with usability, performance, and even functionality. For example, if usability concerns are not addressed in the design of a secure system, users respond by circumventing security mechanisms [1, 2]. Achieving a balance between the intrusiveness of security mechanisms [3] and usability goals is an important consideration in designing successful secure software systems. Security goals can have their own contradictions because confidentiality, integrity, privacy, accountability, availability, and recovery from security attacks often conflict fundamentally. For example, accountability requires a strong audit trail and end-user authentication, which conflicts with privacy needs for user anonymity [3]. 
Ultimately, security is about balancing the trade-offs among the competing goals of multiple actors to achieve a "good enough" security. In current practice, security designers often adopt security mechanisms such as firewalls, access control, or encryption without explicit recognition of, and systematic treatment of competing design objectives originating from various stakeholders. While risk assessment methods such as $[4,5]$ address balancing the costs and effects of security solutions to achieve good-enough security, assuming security costs and benefits are measurable, this paper focuses on qualitatively analyzing trade-offs that security goals and alternative security solutions impose on other quality objectives.

This question then arises: what conceptual modeling techniques can be used to help designers analyze security trade-offs to achieve "good enough" security? To our knowledge, existing conceptual modeling techniques for modeling security-related information and trade-off analysis techniques do not raise and answer this question.

The remaining parts of this paper are structured as follows. In Section 2, we consider the criteria for a suitable conceptual modeling technique for dealing with security trade-offs. In Section 3, a number of existing approaches to security trade-off analysis are reviewed and compared to the introduced criteria. From analyzing the limitations of existing methods, in Section 4, we propose a conceptual modeling technique for modeling and analyzing security trade-offs in a multi-actor setting. The meta-model of security concepts is introduced as well. In Section 5, we describe the goal model evaluation and trade-off analysis technique. Section 6 summarizes the results of some case studies. Finally, Section 7 discusses the results and limitations of the approach.

\section{Conceptual Modeling Criteria for Security Trade-offs Analysis}

Trade-off analysis is a systematic examination of the advantages and disadvantages of requirements and/or design choices for a system to achieve the right balance among several competing goals [6]. When some goals are not sufficiently satisfied, designers need to explore further alternatives that can better achieve those goals, without detrimentally hurting the others. Each potential solution can have positive effects on the achievements of some goals while having negative effects on others. A careful and systematic process for security trade-off analysis can be very challenging, because to resolve security trade-offs one need to consider competing goals of multiple stakeholders, risk of attacks, security countermeasure, and their impacts.

In this context, we ask: what are the criteria for a proper conceptual modeling technique for dealing with security trade-offs? What concepts need to be modeled, and how are they involved in the trade-off analysis? In many engineering disciplines, trade-offs are analyzed using detailed mathematical models. However, in designing secure software systems, multiple stakeholders with diverse, incommensurable, and competing goals impose security trade-offs that cannot be reduced easily to mathematical functions. Conceptual modeling techniques, on the other hand, offer the possibility of analyzing the factors that contribute to trade-offs and their structural compositions and relationships. 
The conceptual foundation for trade-off modeling needs to convey the idea that attempting to improve one quality can adversely affect other quality objectives. Therefore, the conceptual modeling technique needs to provide conceptual constructs to express 1) Design goals and objectives, alternative operationalizations to achieve the objectives, and the impacts of alternative operationalizations on goals and solutions; 2) Actors who seek alternative design solutions to achieve their individual goals and objectives. Furthermore, security objectives are not affected only by alternative security solutions and operationalizations of quality goals. In case of security goals, the trade-off constructs need to express; 3) Threat of external/internal actors and vulnerabilities that impact security and other requirements.

1) Design Goals and Objectives: Security and other trade-offs take root from conflicts among design objectives that originate from stakeholder goals. While selecting a security solution among alternatives is difficult, the more fundamental problem is that designers need to decide about alternate security mechanisms subject to multiple factors such as cost, time-to-market, various non-functional requirements (NFRs), security policies, standards, and individual goals of various stakeholders. Therefore, the conceptual foundation for expressing trade-offs needs to provide means for modeling stakeholders' goals and design objectives. By modeling the alternative solutions that operationalize the objectives and structuring the impact of operationalizations on the goals, one can analyze what causes trade-offs among objectives. To resolve the trade-offs, the model needs to express the extents to which design objectives are satisfied or denied. The extents or measures could be quantitative or qualitative. Quantitative approaches can greatly simplify decision making, but can be difficult to apply due to lack of agreed metrics, subjective quality requirements, or unavailability of accurate measures in the early stages of the system development. The modeling technique should be able to support analysis despite inaccurate, incomplete, or subjective knowledge about goals as well.

2) Actors: Design objectives typically originate from multiple sources and stakeholders such as the system's users, administrators, top managers, project managers, and customers. The conceptual modeling technique needs to consider multiple actors that impose competing goals on the designer. The modeling technique should be able to model trade-offs that occur within a single actor or across multiple actors.

3) Security Specific Concepts: Security requirements are needed because of the threat of malicious actors. Achievement of security objectives are affected by threats of internal or external actors and existence of vulnerabilities in system design. Tradeoffs cannot be resolved without relating the impacts of attacks and exploitation of vulnerabilities on the systems functionality and quality objectives. Therefore, the conceptual modeling technique for modeling security trade-off needs to model security specific concepts such as threats and vulnerabilities. In addition, decision makers need an expression of the security benefits of the solutions [7] to compare their trade-offs. Hence, the modeling technique should provide means to model to what extent attacks are successful, how attacks affect stakeholders' and design goals, and how countermeasures protect the stakeholders' goals against the threats. 


\section{Existing Approaches to Security Modeling and Trade-offs}

Security requirements engineering frameworks derive security requirements based on security-specific concepts such as assets, threats, and vulnerabilities, taken from the security engineering domain. To incorporate security-related aspects into the requirements engineering and software design processes, different approaches tackle the security requirements from different viewpoints.

Attack Modeling Techniques. Some approaches such as Attack Tree [8] and Attack Graph [9] focus on modeling attacks and adversaries. Approaches such as misuse cases invert the normal functionalities of the system to express the misuses and malicious behavior. As proposed by Sindre and Opdahl [10], a misuse case is a use case from the point of view of a hostile actor which poses a threat to the system. They claim that looking at systems from a misuser perspective increases the chance of discovering threats that would otherwise be ignored.

The misuse case approach is adopted by Alexander [6] for trade-off analysis. Alexander asserts that the misuse case approach accurately visualizes the structure of the situation in a way that emphasizes the essential points of conflicts and trade-offs; therefore, use/misuse case representation may make trade-off analysis and human judgments more informed and systematic. Another similar approach is the notion of abuse case, proposed by McDermott and Fox [11], which helps to model a type of interaction between a system and actors where the results of the interaction are harmful to the system.

Another example of inverting normal behavior for analyzing security attacks is the notion of anti-goals. Van Lamsweerde [12] proposes a goal-oriented framework for generating and resolving obstacles to achieve goals. The malicious obstacles, called anti-goals, are set up by the attackers to threaten security goals. The anti-goals are refined to form a threat tree, in which the leaf nodes are either software vulnerabilities or anti-requirements. New security requirements are then obtained as countermeasures.

Security Goals and Requirements Modeling. Some approaches focus on modeling security-related information, security requirements, and security goals. The UMLsec modeling language [13] is an example of a security-specific conceptual modeling approach for modeling security requirements and security-specific information. The main uses of this approach are first, to encapsulate knowledge and make it available to developers in the form of a widely-used design notation, and secondly, to provide formal evaluation to check if the constraints associated with the UMLsec stereotypes are fulfilled in a given specification.

SecureUML is another UML-based modeling language for the model-driven development of secure distributed systems. SecureUML takes advantage of RoleBased Access Control (RBAC) for specifying authorization constraints by defining a vocabulary for annotating UML models with information relevant to access control [14]. The SecureUML meta-model is defined as an extension of the UML metamodel, and the concepts of RBAC are represented directly as meta-model types.

Goal and Agent Oriented Modeling and Analysis. In addition to attacks and protection aspects, security requirements are derived from analysis of interactions and 
dependencies of social actors in the organizational contexts [17, 18]. Such considerations to derive security requirements bring other related issues such as trust, ownership, permission and delegation among actors to the security requirements modeling and analysis process [19, 20].

Agent and goal oriented approaches describe systems as intentional agents that depend on each other or compete to achieve their goals. In recent years, such approaches in requirements engineering have emerged for analyzing and designing complex information systems. Examples of goal oriented frameworks are KAOS [21] and the NFR framework [22]. Agent oriented approaches include the $i^{*}$ [23] and Tropos [24] frameworks. Several approaches such as [17, 18, 19, 25, 26, 27] propose frameworks for modeling and analyzing security concepts by taking advantage of agent and goal oriented techniques. The majority of these approaches employ qualitative goal evaluation, while [25] suggests a quantitative approach for analyzing security requirements.

Risk Analysis and Decision Making. Risk analysis has been considered as an important activity of security requirements engineering and secure software design. The risk-based security requirements engineering framework in [15] is concerned with integrating requirements engineering practices with security engineering and interleaving requirements and architecture design by adopting risk analysis practices as a key activity. The approach aims to analyze the impacts of threats on the business by linking the risk impacts with the business value.

The CORAS framework [4] provides another UML profile for risk assessment. The proposed profile defines UML stereotypes and rules for specialized UML diagrams to support of model-based risk assessment. Asset diagram is proposed to identify assets; threat diagram captures unwanted incidents causing loss in asset value. State analysis diagram is proposed to estimate and document the frequency and consequence of the unwanted incidents, and treatment diagrams are models extended with specialized use cases representing treatments.

Another approach for deciding on security requirements and design is proposed in [16], where probabilistic inference on security influence diagrams and Bayesian Networks are used to support trade-off analysis. In this work, authors discuss the criteria of the language for security requirements modeling and analyzing. They assert that the language should be able to represent decision's maker goals, domain of control, and causal relation between goals (indirect and controlled).

The above survey of security-related modeling notations and security requirements frameworks reveals that existing security conceptual modeling techniques do not consider trade-offs between security and other quality objectives explicitly. While trade-off analysis is considered in the misuse case approach of Alexander [6], use/misuse cases do not express quality requirements, their operationalizations, and impacts. More generally, trade-off analysis has been considered in a few software engineering research efforts. Trade-offs are mostly considered between alternative architecture and design solutions in contributions such as Architecture Tradeoff Analysis Method (ATAM) [28] and Cost-Benefit Architecture Analysis (CBAM) [28], Aspect-Oriented Risk-Driven Development (AORDD) [29], and Security Verification and security solution Design Trade-off analysis (SVDT) [7]. Although 
these approaches do not provide a conceptual modeling framework for expressing trade-offs, studying the decision making process in these approaches is of interest for extracting the criteria for a conceptual modeling framework that enables trade-off analysis.

In what follows, we study Architecture Tradeoff Analysis Method (ATAM) and Cost Benefit Analysis Method (CBAM) [28] as general purpose and widely used architectural trade-off analysis methods which consider security as one of the quality scenarios. SVDT and AORDD are studied as security-specific methods and the representatives of quantitative analysis methods based on probabilistic reasoning. Since the proposal for security trade-offs modeling and analysis in this paper is based on expressing competing goals of multiple actors, we finally study whether the existing agent and goal oriented approaches provide the required conceptual modeling basis for expressing trade-offs.

\subsection{ATAM and CBAM essence}

Bass et al. [28] introduce a framework for modeling quality attributes and architectural alternative designs using the notion of scenarios and tactics respectively. A quality attribute scenario is a quality-attribute-specific requirement, and consists of six parts: Source of stimulus, Stimulus, Environment, Artifact, Response, and Response measure. Achievement of quality scenarios relies on tactics.

ATAM is a labor-intensive evaluation method to analyze whether an architecture decision satisfies particular quality goals. ATAM helps designers to prioritize scenarios and evaluate alternative tactics using a "Quality Attribute Utility Tree". Scenarios that have at least one high priority of importance or difficulty are chosen for a detail analysis to examine if the selected tactics satisfy the scenario.

The result of the ATAM analysis is an "Architectural Approach Analysis" table for each quality scenario. In this table, evaluators identify and record sensitivity, tradeoff, risks and non-risks points of alternative tactics for satisfying a scenario. Sensitivity and tradeoff points are architectural decisions that have effect on one or more quality attributes, the former positively and the latter negatively. In ATAM, a risk is defined as an architectural decision that may lead to undesirable consequences. Similarly, a nonrisk is an architectural decision that, upon analysis, is considered safe. The conceptual elements related to trade-offs in ATAM may be captured in a meta-model as in Fig. 1.

CBAM [28] picks up where ATAM leaves off. It takes ATAM outputs as input and adds cost factors to the trade-off analysis. CBAM uses scenarios as a way to concretely express specific quality attributes. Stakeholders vote for scenarios to select the top priority scenarios for further analysis. Stakeholders assign the value for calculating stimulus-response utility per each scenario, which helps to create the utility-response curves. The utility is based on the importance of each scenario and its response value. The overall utility is combined with the project cost of an architectural strategy to calculate the final Return On Investment (ROI) measure. The ROI value for each architectural strategy is the ratio of the total benefit to the cost. Using the ROI value, the architectural strategies can be rank-ordered and compared, and a final decision made. 


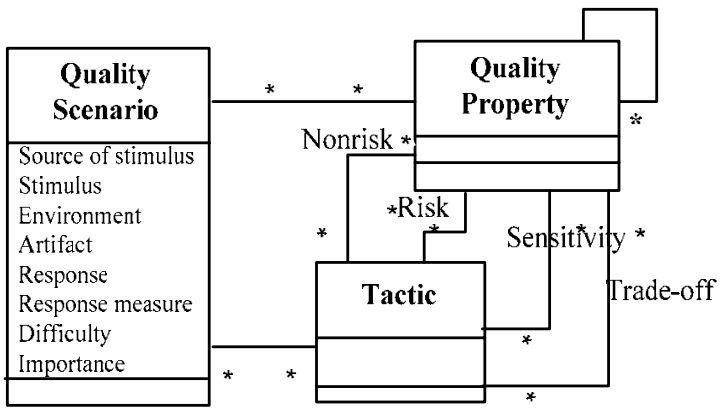

Fig. 1. A Meta-model of trade-off elements in the ATAM.

\subsection{SVDT and AORDD Approach}

Houmb et al. [7] propose the Security Verification and security solution Design Trade-off analysis (SVDT) approach using UMLsec for modeling security solutions and Bayesian Belief Network (BBN) as the basis of trade-off analysis implementation. UMLsec is used to verify if the design solutions satisfy the security requirements. Design solutions that pass the verification are then evaluated using security solution design trade-off analysis.

The SVDT approach is a complementary work on Aspect-Oriented Risk-Driven Development (AORDD) framework [29]. AORDD framework provides risk assessment process and cost-benefic trade-off analysis. Both SVDT and AORDD trade-off analysis methods compute Return on Security Investment (RoSI) using BBN. Fig. 2 illustrates the relationship between the main concepts involved in AORDD risk assessment, which specifies the structure of the inputs to the AORDD cost-benefit trade-off analysis and the topology of the BBN.

The result of risk assessment is a list of misuses which need security treatments. This list and a list of alternative security treatments are fed into the BBN to compute the RoSI. RoSI is computed using a set of trade-off parameters, such as priorities, security risk acceptance criteria, standards, laws and regulations, policies, business goals, budget, and time-to-market. RoSI for a particular solution is derived by evaluating the effect and cost of the solution against the security requirements, and the misuse impact and frequency [7]. 


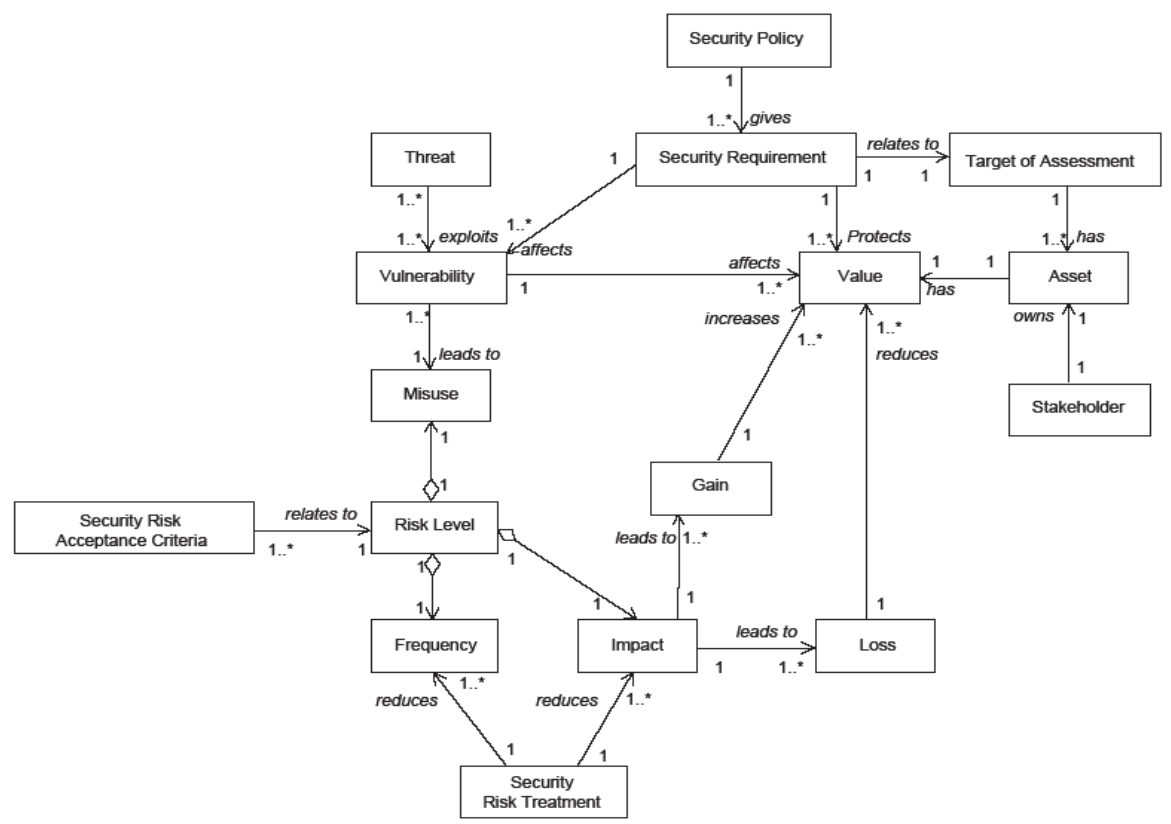

Fig. 2. ARODD risk assessment main concepts and relation [29]

\subsection{Secure Tropos/i*}

The proposed approaches in [17, 18, 19, 26, 27] take advantage of the $\mathrm{i}^{*}$ and Tropos frameworks for modeling and analyzing security requirements. In these approaches, systems are modeled as intentional agents collaborating or competing with each other to achieve their goals. Security issues arise when some actors, while striving to achieve their own goals, intentionally or unintentionally threaten other actors' goals; therefore, agent and goal oriented approaches provide a suitable basis for dealing with security.

The approach in [17] suggests using relationships among strategic actors for analyzing security requirements. In [17], potential attackers of the systems are distinguished from other actors. Liu et al. [18] analyze attackers, dependency vulnerabilities among actors, countermeasure, and access control using the $\mathrm{i}^{*}$ modeling framework. In [19], a framework known as Secure Tropos for modeling and analyzing security requirements based on the notions of trust, ownership, and permission delegation is developed. In [26, 27], the "threat" and "security constraint" modeling elements are added to the $i^{*}$ meta-model. Threat elements are employed in the "security diagram" to express potential violation against the security goals, and security constraints are used to impose security requirements on actors' dependencies. The meta-model of related concepts to the Tropos goal model, which is the core of all these approaches, is depicted in Fig. 3. 


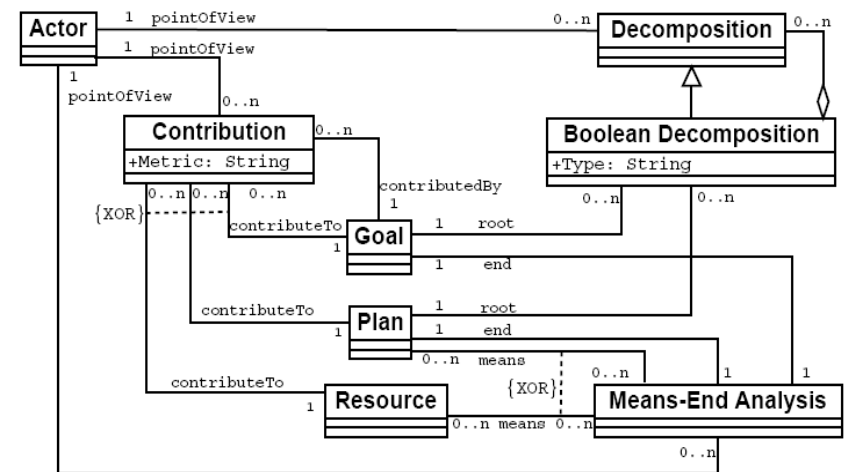

Fig. 3. Part of Tropos meta-model for goals and related concepts [30]

\subsection{Discussion and Limitations of the Surveyed Approaches}

ATAM and CBAM provides a labor-intensive method for deciding on alternative architecture design, and AORDD and SVDT are designed for comparing alternative security solutions based on the solution costs and benefits, potential misuse effects and probability, and trade-off parameters. In these two groups of work, trade-offs are expressed at different levels of explicitness. In the ATAM and CBAM approaches, trade-offs are listed in a table rather than expressed by models, formulas, or a computation mechanism. Trade-offs are analyzed by an architecture expert who evaluates each design tactic in terms of its costs and benefits. In the AORDD and SVDT methods, the trade-off model is developed by the BBN designer instead of the target system designers and analysts. In this way, a single fixed formulation of relationships between trade-off parameters are reused for multiple projects.

In the ATAM approach, trade-offs among quality scenarios and tactics in the "Architectural Approach Analysis" table are expressed indirectly and implicitly. In this table, for each scenario alternative tactics are analyzed. However, the impacts of tactics are analyzed on one specific scenario rather across multiple scenarios. Moreover, ATAM does not incorporate the degree of sensitivity or trade-off between tactics and qualities in the analysis procedure, since the "Architectural Approach Analysis" table does not provide means for expressing the impact of tactics on stimuli (attacks) and does not specify the consequences of applying a solution on other quality scenarios. The ATAM process does not separately capture the impact of each tactic on stimuli of security scenarios (attacks).

ATAM does not consider cost as a trade-off factor that usually has the highest priority over the other factors. CBAM is proposed to address this weakness. The CBAM approach to trade-off analysis requires the designer to obtain accurate quantitative measures of current response and expected response of architectural strategies for each affected scenarios. The major obstacle toward applying CBAM would be unavailability and inaccuracy of such measures in the early stages of development and lack of agreement on metrics and measurement methods. 
CBAM relies on the utility values gathered from votes of various stakeholders through consensus. In this way, CBAM resolves the conflict of goals among stakeholders by computing the ROI of each strategy based on their votes for utility of each strategy. However, the accuracy and reliability of the utility values and stakeholders' confidence in their votes is an issue in validity of CBAM analysis. Finally, the ATAM and CBAM methods are not designed for security trade-off analysis and the impact of other forces such as attacks and vulnerabilities are not explicitly incorporated into the analysis.

SVDT and AORDD are specifically designed for making security trade-offs, relying on a pre-defined probabilistic reasoning model of trade-offs in the form of a BBN. This requires the software designers to obtain quantitative measures or qualitative scales of the impacts of the misuses and solutions. The major limitation is the inaccuracy or unavailability of quantitative data on the impact of misuses and solutions especially in the early stages of the development lifecycle.

Generally, the suggested BBN topologies in [7] do not consider a general source of trade-off inputs such as NFRs and functionalities. The single value of RoSI does not indicate what quality objectives are achieved or failed. The input trade-off parameters of the designed BBN are limited to factors such as budget, time-to-market, laws and regulation, which had been designed and incorporated into the BBN topology. It is assumed that a single topology that is designed for trade-off analysis would be applicable in every context.

The final result of the trade-off analysis is a number (RoSI), indicating the return on investment for each security solution. Although automatic computation of RoSI using the trade-off inputs and parameters fed into the BBN is efficient and desirable, the designers and analysts do not have explicit traces to follow what aspects of the design caused the difference in the final result of RoSI. In other words, the only metric to compare two alternative security solutions is the RoSI values for those solutions, which makes it difficult to judge the impact of multiple contributing factors of alternative different design solutions. In addition, if the system designers and analysts need to trace the impact of changes in BBN parameters, they need to learn about the topology of the BBN which they have not designed.

Table 1 summarizes a comparison of the ATAM/CBAM and AORDD/SVDT approaches based on the evaluation criteria discussed in section 2 . The third column compares the conceptual modeling constructs that agent and goal oriented approaches provide for expressing trade-offs among design objectives.

Agent and goal oriented approaches such as [17, 18, 19, 26, 27] provide the conceptual basis for security trade-off modeling and analysis. However, a mechanism for security trade-offs analysis is not elaborated in these frameworks. The method in [18] lacks a direct and explicit way to model the competition among malicious and non-malicious actors' goals. In this approach, trade-off modeling among goals is limited to the non-malicious actors. The proposed framework in [19] does not support modeling malicious behavior. In [26, 27], threats are modeled in the security diagram, but the model does not trace security goals or threats to the source actors. This approach does not consider modeling the relation between countermeasures and 
attacks. Although security trade-offs can be expressed by the goal oriented methods, the approaches overviewed do not consider security trade-offs explicitly.

Table 1. A comparison of existing approaches based on the criteria of the conceptual modeling technique for security trade-off analysis

\begin{tabular}{|c|c|c|c|}
\hline $\begin{array}{c}\text { Method/ } \\
\text { Requirements }\end{array}$ & АТАМ/СВАМ & SVDT/AORDD & i*/Tropos \\
\hline $\begin{array}{c}\text { Design objectives } \\
\text { and goals }\end{array}$ & $\begin{array}{c}\text { Expressed in terms of } \\
\text { scenarios }\end{array}$ & $\begin{array}{l}\text { Considered by fixed } \\
\text { BBN parameters }\end{array}$ & Modeled by goals \\
\hline $\begin{array}{c}\text { Relations between } \\
\text { objectives }\end{array}$ & $\begin{array}{c}\text { Not considered } \\
\text { explicitly }\end{array}$ & $\begin{array}{c}\text { Considered in the fixed } \\
\text { BBN topology }\end{array}$ & $\begin{array}{c}\text { Modeled by } \\
\text { contribution links }\end{array}$ \\
\hline $\begin{array}{l}\text { Alternative design } \\
\text { solutions }\end{array}$ & $\begin{array}{l}\text { Expressed in terms of } \\
\text { tactics }\end{array}$ & $\begin{array}{l}\text { Each alternative security } \\
\text { solution is analyzed by } \\
\text { the UMLsec reasoning } \\
\text { and BBN cost benefit } \\
\text { analysis }\end{array}$ & $\begin{array}{c}\text { One goal model } \\
\text { can express and } \\
\text { evaluate } \\
\text { alternative design } \\
\text { solutions } \\
\end{array}$ \\
\hline $\begin{array}{c}\text { Impacts of } \\
\text { objectives and } \\
\text { solutions on each } \\
\text { other }\end{array}$ & $\begin{array}{l}\text { Utility tree in ATAM } \\
\text { doesn't capture the } \\
\text { contributions of } \\
\text { scenarios on each other/ } \\
\text { CBAM considers by } \\
\text { utility and side effects } \\
\text { of an architectural } \\
\text { strategy }\end{array}$ & $\begin{array}{l}\text { Encoded in the BBN } \\
\text { topology }\end{array}$ & $\begin{array}{c}\text { Modeled by } \\
\text { contribution links }\end{array}$ \\
\hline $\begin{array}{c}\text { Extents of } \\
\text { objectives' } \\
\text { achievement }\end{array}$ & $\begin{array}{l}\text { Not considered in } \\
\text { ATAM/ Expressed in } \\
\text { terms of Utility- } \\
\text { Response Curves in } \\
\text { CBAM }\end{array}$ & $\begin{array}{l}\text { Expressed by parameters } \\
\text { fed into the BBN and } \\
\text { mostly quantitatively }\end{array}$ & $\begin{array}{c}\text { Expressed } \\
\text { qualitatively by } \\
\text { goal model } \\
\text { evaluation labels }\end{array}$ \\
\hline $\begin{array}{l}\text { Inaccurate or } \\
\text { incomplete } \\
\text { knowledge }\end{array}$ & Is not dealt with & $\begin{array}{c}\text { In terms of probability } \\
\text { density functions (pdf) in } \\
\text { BBN } \\
\end{array}$ & $\begin{array}{c}\text { Expressed by } \\
\text { contribution type } \\
\text { Unknown }\end{array}$ \\
\hline Multiple actors & $\begin{array}{c}\text { Expressed implicitly by } \\
\text { multiple stimuli sources } \\
\text { in ATAM. In CBAM } \\
\text { multiple stakeholders } \\
\text { votes are taken into } \\
\text { account } \\
\end{array}$ & $\begin{array}{l}\text { The BBN topology can } \\
\text { contain parameters from } \\
\text { multiple actors' point of } \\
\text { interest }\end{array}$ & $\begin{array}{l}\text { In terms of } \\
\text { agents, actors, } \\
\text { roles, and } \\
\text { positions }\end{array}$ \\
\hline $\begin{array}{l}\text { Security specific } \\
\text { forces }\end{array}$ & Not considered & $\begin{array}{l}\text { Some concepts are } \\
\text { modeled }\end{array}$ & $\begin{array}{l}\text { Some concepts } \\
\text { are modeled }\end{array}$ \\
\hline
\end{tabular}

\section{The Security Trade-offs Modeling Notation}

We propose a meta-model of security concepts for systematically addressing security trade-offs, considering the limitations of existing approaches and reviewing well known security knowledge sources such as NIST guidelines, standards like [31], CERT [32], and widely used textbooks such as [33, 34]. Fig. 4 depicts the meta- 
model of security concepts where the core concepts are actors and goals. Actors employ mechanisms or attacks to achieve their goals or malicious goals, protect assets, patch or exploit the vulnerabilities.

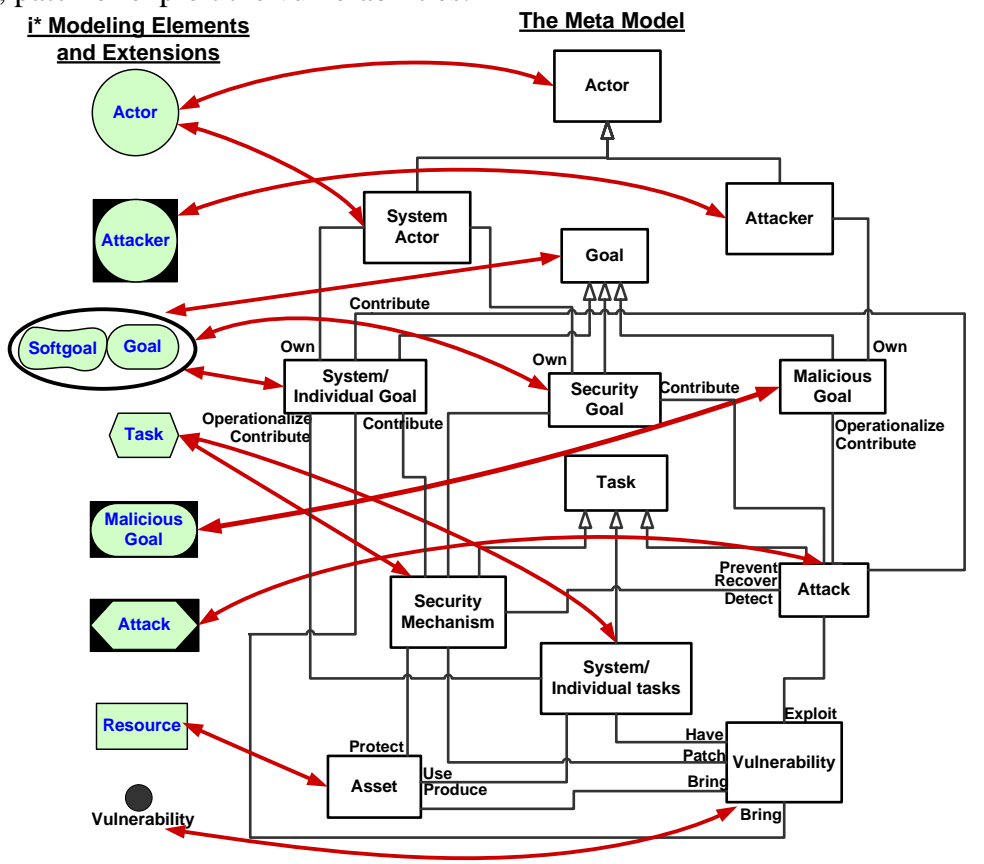

Fig. 4. Meta-model for the security concepts used in the proposed conceptual modeling technique and their mapping to the i* framework elements.

The proposed modeling notation for expressing security trade-offs builds upon the $i^{*}$ framework which provides a notation to model actors, their goals and intentional dependencies and competitions among the actors. Actors achieve goals on their own or depend on each other for goals to be achieved, tasks to be performed, and resources to be furnished. Quality goals, which do not have clear-cut criteria for satisfaction degree, are modeled as softgoals. Means-ends relation between goals and tasks is used to model alternative ways to achieve a goal [35]. Contribution links enable expressing the impact of tasks and (soft)goals on other softgoals. However, the $i^{*}$ notation lacks explicit modeling constructs for concepts such as attacks and vulnerabilities. In this section, we propose some extensions to the $i^{*}$ notation, which provide conceptual structures for modeling and analyzing security trade-offs. Fig. 4 shows how elements of the meta-model are mapped to the $i^{*}$ modeling constructs. In what follows, detailed descriptions of the meta-model elements and corresponding $i^{*}$ modeling elements is given. 


\subsection{Malicious Actor, Goals and Tasks}

Actors depend on, or compete with each other to achieve their goals. Meanwhile, malicious actors try to achieve their own goals. Representing a malicious actor with a different modeling construct in $i^{*}$ was first employed in [17] by highlighting them with a black shadow rectangle. This notation was used to model malicious goals in [18]. We make use of this notation in which malicious goals, softgoals, tasks, and actors are highlighted by a black shadow rectangle. By distinguishing malicious modeling elements from non-malicious ones, we emphasize studying the attackers' goals and tasks. Although attacker's behavior might be partially unknown and generic, an important aspect of trade-off analysis depends on studying attackers' options and the risks they pose to other actors' goals.

A security threat is any malicious behavior that interferes with the achievement of other actors' goals. For example, in Fig. 5, Malicious Employee is the malicious actor whose goal is to Commit a fraud under someone else's name, either through the local network or over the Internet.

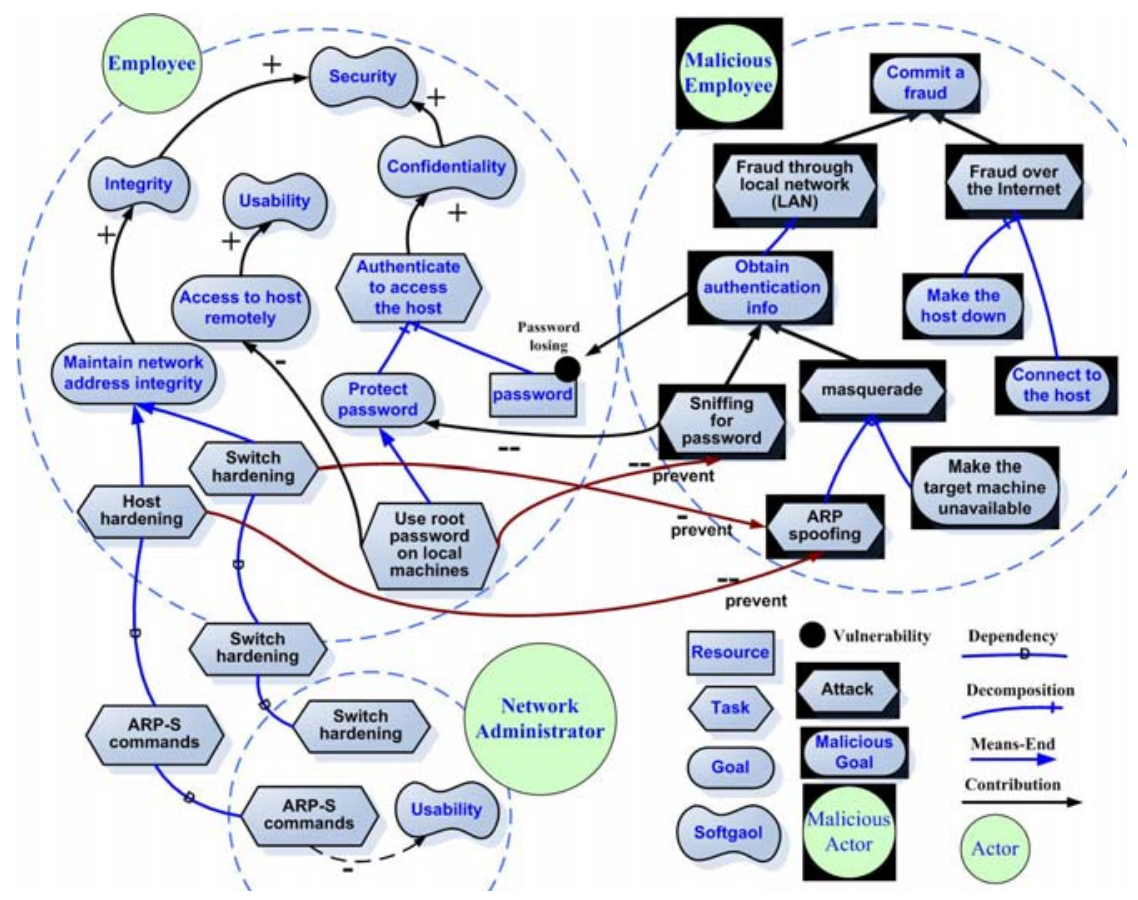

Fig. 5. Example of a multi-actor system modeled using the proposed notation 


\subsection{Assets and Vulnerabilities}

An asset is any thing that has a value for the organization [34]. Physical resources, information, and people can be counted as assets. In this way, the concept of asset is well matched with the "resource" modeling element of the $i^{*}$. Assets can be the services an organization offer or receive, and in this case, can be represented by tasks or goals that actors offer to the "depender" actors.

In security terminology, a vulnerability is any weakness in, or back door to the system [34]. For example, it is said that buffer overflow and password cracking are the most common vulnerabilities of many computer systems [33]. Generally, assets or services bring vulnerabilities to the system, and attackers usually try to achieve malicious goals through exploiting a vulnerability. In the i* notation, tasks are usually decomposed to goals, softgoals, other tasks, and resources. In this way, the harm from an attack is the cost of the failed task that relies on the compromised assets. In a similar approach in [36, 37], threats are described in terms of assets, the action that exploits the assets, and the subsequent harm.

Although vulnerability that arises from dependencies among actors is a fundamental concept in the $i^{*}[18], i^{*}$ does not provide modeling construct to express vulnerabilities. We add the vulnerability modeling element to $i^{*}$, accompanied with a graphical representation. For example, in Fig. 5, to protect confidentially employees are authenticated by the host. Hence, Password is one of the employees' assets they need to protect. On the other hand, Password losing is one of the most important vulnerability points in computer systems. Sniffing for password is an attack against the goal of Protect password. Through this attack and Password losing vulnerability, the goal of Fraud under someone else's name can be satisfied, and the attacker gains a valuable asset: the Password.

\subsection{Relationship between Attacks and Security Mechanisms}

In the $i^{*}$ notation, the relationship between softgoals and other elements is modeled by contribution links [23]. If an element has a negative impact on a softgoal, yet not enough to prevent it, the contribution link type is "hurt" and visualized by "-“. If the element is sufficient to prevent a softgoal, the contribution link type is called " break" and represented by "--". This qualitative approach is used to model the impact of attacks on softgoals and the impact of security mechanisms on malicious tasks and goals. In security engineering, various mechanisms have different effects on attacks. Contribution of mechanisms to attacks are categorized as 1) Prevent 2) Detect 3) Recover [34].

These categories are added as attributes of the contribution links. "Detect" and "Recover" contribution links may partially mitigate the effect of attacks. To sufficiently counteract an attack, security mechanisms must be related to the attack with a "Prevent" contribution link. Mechanisms related to the attacks with Detect contribution links cannot control any attack. Similarly, Recover contribution links is used to indicate that the mechanism recovers the system after the attack. Recover link would be useful to express availability and integrity goals that rely on recovering the system after the failure. 


\subsection{Expressing Trade-offs using the Proposed Conceptual Structure}

The proposed approach provides the means to model goals and trace them back to the source actors. In this approach, trade-offs among goals are modeled by contribution links. Through contribution link types of -, --, + and ++ [22], the qualitative effect of alternative solutions are propagated to the other goals and attacks. The $i^{*}$ notation offers the conceptual structure to model trade-offs between sub-goals of high level goals. For example, in Fig. 5, the employee can Use root password on local machines to completely prevent the attack of Sniffing for password [33]. However, this security solution contributes negatively to the Access to host remotely goal, and it has negative influence on the Usability softgoal consequently. In this way, the trade-off among usability and security is modeled through relationships among the corresponding refined sub-goals.

\section{Trade-off Analysis and Decision Making}

In the previous section, we proposed a conceptual modeling technique for modeling security trade-offs. In this part, we propose a trade-off analysis method for use with the trade-off model. Designers need to balance the trade-offs to mitigate the security risks and yet satisfy the goals of multiple actors. A goal is at risk when it may be denied (partially or fully) by the successful behavior of malicious actors. Partially or fully denial of goals are the result of contribution links of type "-" and "- -". Designers need to examine available alternative security solutions, and verify the impacts of each one on attacks and goals to finally select the one which fits with goals of multiple actors. Goal model evaluation is the procedure to ensure that actors' top level goals are satisfied by the choices they have made [38]. The security goal model evaluation, which consists of interactive qualitative reasoning, is based on the method proposed in [22] and refined in [38]. Fig. 6 depicts the proposed security trade-off analysis procedure.

In the first step, evaluator assumes that attackers are successful in performing tasks and satisfying their goals, since attackers are usually external actors that designer has no sure knowledge of their abilities and skills. Therefore, the leaf nodes in attackers' goal model are labeled fully satisfied. This assumption does not imply that the risk of attacks is definite, as it is possible that evaluation of attackers' goal model yields to denial of higher goals of attacker. The leaf labels are propagated to upper goals. Once the impact of malicious actors' behavior is propagated to the entire goal model, the evaluator assigns labels to the tasks and goals that operationalize security mechanism (step 7). This label indicates the evaluator's judgment about the success of the corresponding actor in performing a security task or achieving a security goal. This judgment could be based on knowledge of previous experiences, empirical studies, or subjective knowledge [7].

In step 9, the goal model indicates which goals are fully or partially satisfied or denied for the examined security solution. The procedure iterates until a security design solution is found that, based on the evaluator's perception, satisfies an acceptable configuration of goals. However, the evaluator may prefer to examine 
further alternatives to select the security design solution that satisfies more goals. After evaluating an alternative, the status of some goals may be unknown, prompting the designer to elaborate the models (step 10). In case of conflict of goals, other alternatives should be examined to resolve the conflicts (step 11).

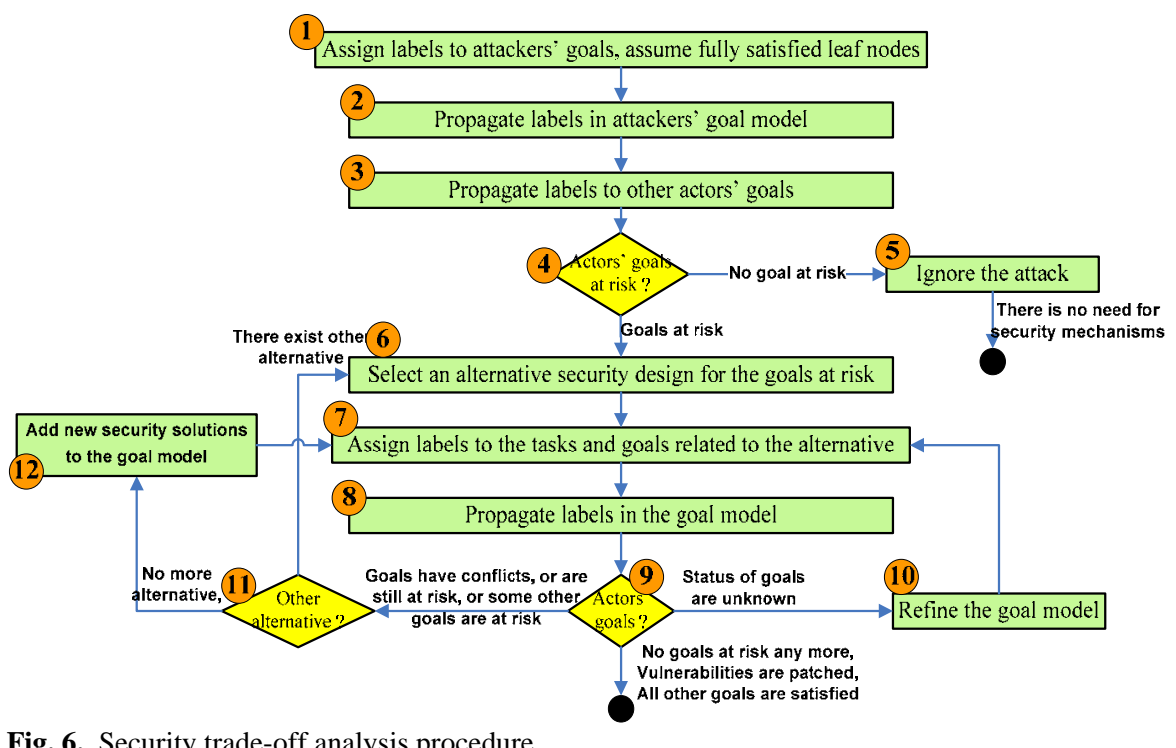

Fig. 6. Security trade-off analysis procedure

Propagation of the labels is based on the contribution types and rules summarized in Table 2. The contribution in [38] provides details about aggregation rules for multiple contributions. The rules provided in Table 2 are merely valid for the Prevent contribution type, as we discussed earlier that recovering from, or detecting an attack do not lead to controlling the attack.

Table 2. Evaluation labels and propagation rules from [22, 38]

\begin{tabular}{|c|c|c|c|c|c|c|}
\hline \multicolumn{2}{|c|}{ Child Node } & \multicolumn{5}{|c|}{ Contribution Type (Prevent) } \\
\hline Label Name & Symbol & ++ & + & - & - & $?$ \\
\hline Satisfied & $\checkmark$ & $\checkmark$ & $\sqrt{ }$ & $x$ & $x$ & ; \\
\hline Weakly Satisfied & $\sqrt{ }$ & $\sqrt{ }$ & $\sqrt{ }$. & $\stackrel{x}{x}$ & $x$ & ; \\
\hline Conflict & 2 & 2 & 2 & 2 & 2 & $?$ \\
\hline Unknown & $?$ & $\dot{q}$ & ; & ; & ? & $\dot{?}$ \\
\hline Weakly Denied & $x$ & $x$ & $x$ & $\checkmark$ & $\checkmark$ & ; \\
\hline Denied & $x$ & $x$ & $\underline{x}$ & $\checkmark \cdot$ & $\checkmark$ & $\dot{q}$ \\
\hline
\end{tabular}




\section{Case Studies}

For developing the extended $i^{*}$ notation, we worked out a number of examples using NIST guidelines [31] and security engineering knowledge in [33]. In addition to those example cases, we applied the notation to two example cases originally used by other authors to illustrate their similar techniques for doing security modeling and analysis. In the first example case, we modeled and analyzed the Guardian Angel (GA) system [39], a patient and physician supporting system, which was modeled and analyzed in [18]. The second case study is eSAP system, an agent-based health and social care system, which was used as the case study system in [25, 26, 27].

The main objective of the case studies is to evaluate and compare existing goal and agent oriented approaches with the proposed method in terms the expressiveness of security models and ability to express security trade-off. The case studies demonstrate how different approaches to modeling and analyzing security aspects would yield different types of analysis and design decisions. In addition, the contribution of current method is distinguished from the previous works. To illustrate the differences, we have borrowed fragments of the original models developed in [25] and [18].

In a third example case, we modeled and analyzed a simple course registration system, using the proposed extensions to the $i^{*}$ and the framework proposed in [28]. In this case study, the proposed trade-off analysis method is compared with the ATAM to study the differences of ATAM procedural analysis and explicit trade-off modeling in the proposed method.

\subsection{Case Study One: Guardian Angel Project}

GA is a patient and physician supporting system using software agents. This information system is centered on the individual patient instead of the provider, in which a set of "guardian angel" (GA) software agents integrates all health-related concerns about an individual. This personal system will help track, manage, and interpret the subject's health history, and offer advice to both patient (in the case study Abby plays patient role) and provider. Patients provide the medical information to a PDA which is connected to other systems such as the GA-Home, GA-Hospital and peer GA-PDA.

The methodological framework for dealing with security requirements in [18] is illustrated with the examples of designing GA software agents. The requirements elicitation process in this approach starts with identifying and modeling actors and their goals and tasks. In parallel, vulnerabilities, malicious actors and intents are identified and integrated into the goal models. Finally, countermeasures for attacks are chosen and added to the models and process ends with countermeasure analysis.

This approach takes advantage of network of Strategic Dependency (SD) to model the dependency among actors and their goals which provides the basis to analyze the opportunities and vulnerabilities. The basic premise for attacker analysis is that all the actors are assumed guilty until proven innocent. In this way, each actor of the goal model can be a potential attacker and is studied in two roles: its regular role and its potential malicious role. 
In vulnerability analysis, each dependency is analyzed as a potential threat against the system. One of the actors in the dependency relation is substituted by its corresponding attacker, and vulnerable dependencies are highlighted with a black shadow rectangle. Fig. 7 depicts the dependency vulnerability analysis in [18]. Fig. 8 illustrates the original attack model of GA system presented in [16]. For each vulnerable dependency, alternative attacks are identified and modeled as tasks that contribute to the malicious goal.

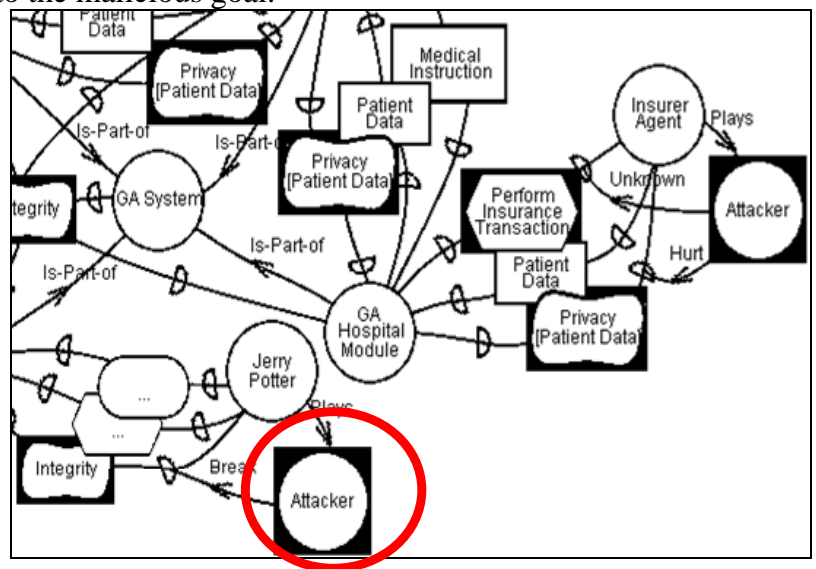

Fig. 7. A part of dependency vulnerability analysis of the GA system, the model is extracted from [18]. Each dependency is examined as a potential threat against the system. Each actor plays role of a potential attacker as well as its regular role

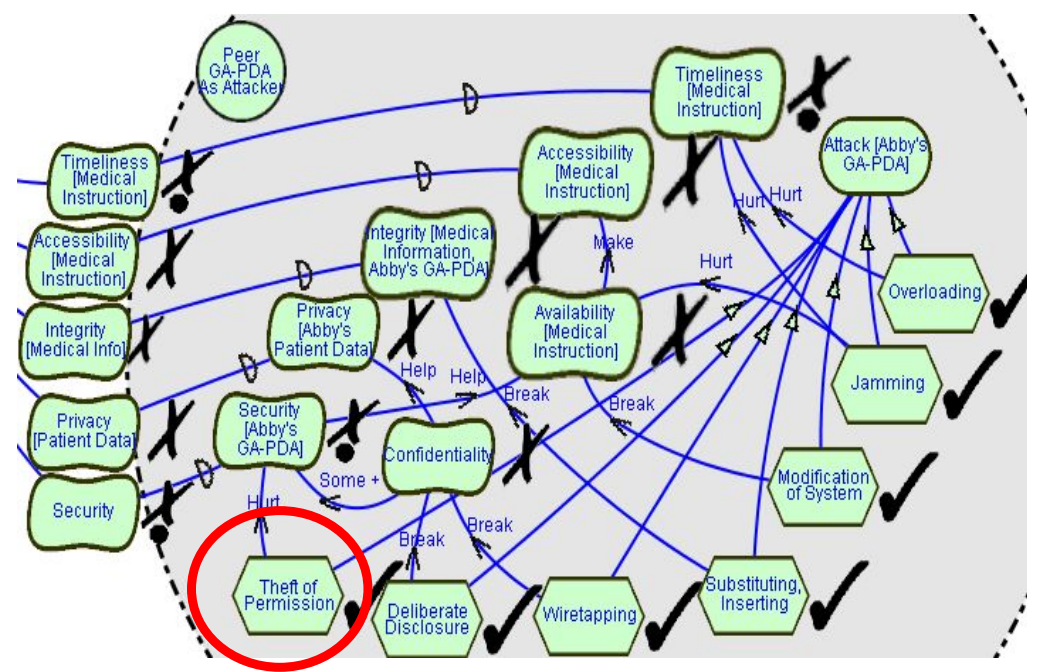

Fig. 8. Threats identification; the original model in [16]. Malicious tasks and affected goals are located inside the boundary of the malicious actor. Malicious goals and tasks are not distinguished from regular goals 
In [18], countermeasures are chosen for each attack. Countermeasures are added to the attacks model, and are related to attacks with a "break" or "hurt" contribution link. Fig. 9 gives the countermeasure analysis model of GA in [18]. The goal model evaluation in original models is limited to the evaluating threats and their corresponding safeguards. The safeguards affect other goals such as performance and usability, but generally, the resulting models in [18] do not capture these trade-offs that security mechanisms impose on other goals.

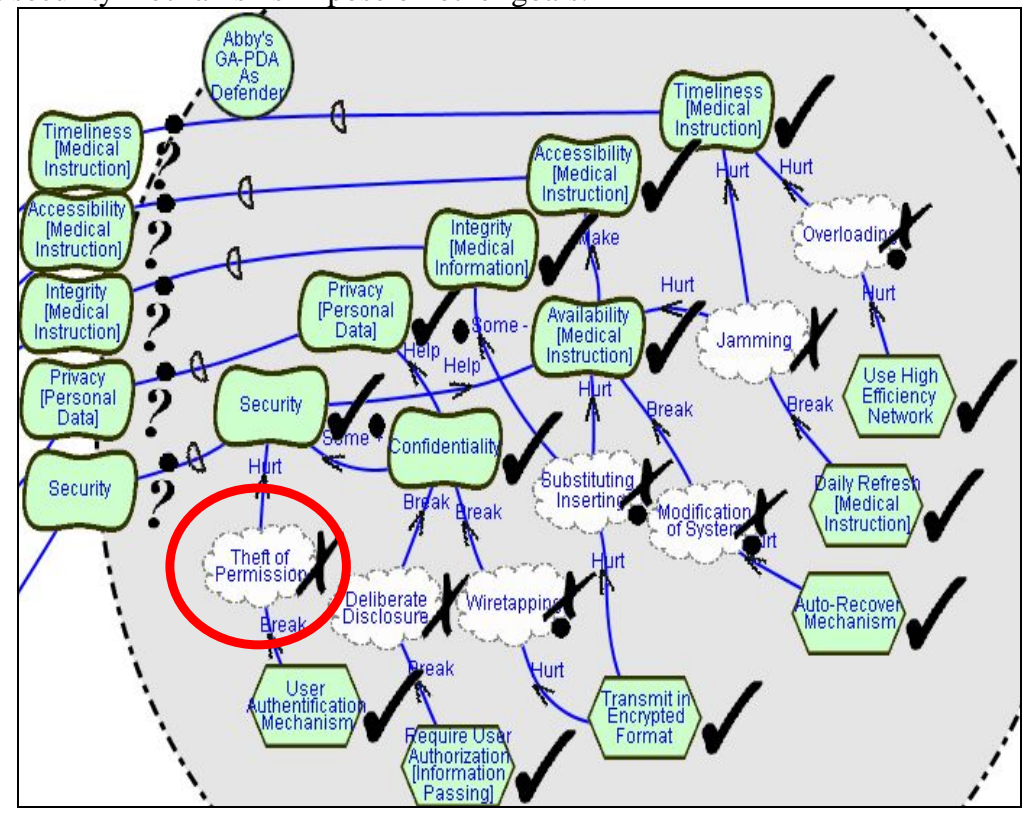

Fig. 9. Countermeasure analysis, original model in [16]. The hypothetical threats are presented as beliefs, since their existence is based on the designer's assumptions. Contributions of countermeasures to the attacks are modeled, but it is not specified which actor employs the security countermeasures and what are the consequences

Fig. 10 depicts the resulting security trade-off model developed using the proposed $i^{*}$ extensions. In this model, the same security mechanisms and goals modeled in Fig. 9 are used to structure the GA-PDA goal model. In this view, potential attacks, malicious goals, and sub-goals are taken into account. This model expressed the impact of on the attacker's goals and the effect of malicious goals and alternative malicious tasks on attacker's goals and softgoals.

Fig. 10 provides explicit model of trade-offs that alternative mechanisms and potential attacks impose. This model provides the designer with a view to potential malicious behavior, alternative protection mechanisms, their trade-offs and consequences. The model provides the security goal model evaluation results as well. The resulting goal model captures the effects of each alternative attack on malicious and non-malicious actors' (soft)goals. As a result, designer can evaluate risk of threats, and select security solutions for attackers' behavior based on the explicit consequence of malicious actors' behavior. The evaluation steps are highlighted by 
number tags. This procedure starts with assigning satisfaction labels to the malicious tasks and goals. For example, in the first step, the evaluator assigns a satisfied label to System's Password Cracking. In the next steps, these labels are propagated through the goal model.

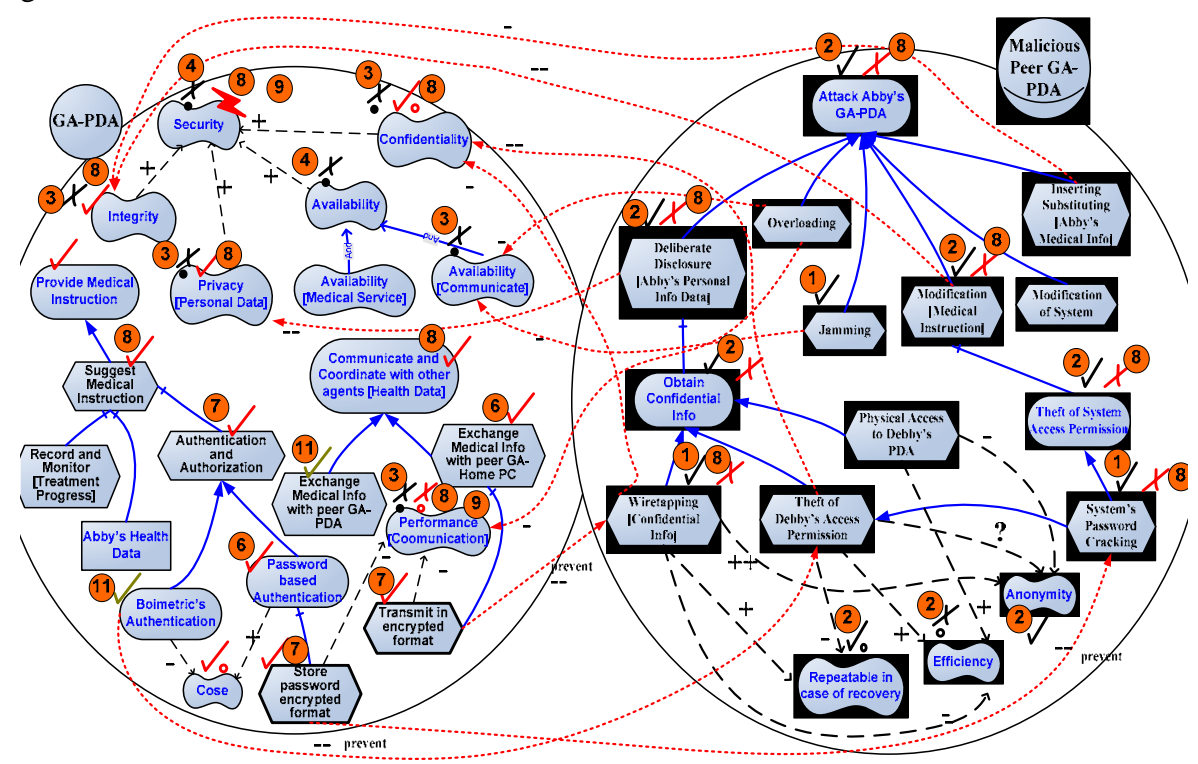

Fig. 10. Security trade-off model and analysis of the GA system, using the proposed extensions. The evaluation method steps are specified by number tags. The right side of the model shows the malicious behavior and its impact on non-malicious side at left.

We now consider the weaknesses in the approach taken in $[18,16]$ and compare it with the proposed approach in the current work. Analyzing the dependencies as the potential vulnerabilities in [18] is useful, but it is not enough to uncover the potential threats imposed by external entities. In addition, the models do not capture goals and intentions of the attacker. The designer needs to know how the attacker may use the vulnerabilities as a backdoor to the system, since deciding among different countermeasures depends on the attacker's goals. For example, the designer needs to differentiate between the goals of a professional hacker and intentions of a curious kid to select proper security mechanisms. The approach in [18] neglects analyzing alternative attacks that each one requires specific security safeguard. In term of expressiveness of conceptual constructs, a major weakness of the modeling approach in Fig. 9 is that both security safeguards and attacks are located inside the boundary of the attacker; therefore, the model does not specify which actor(s) employs the security mechanisms. Finally, the contributions of security mechanisms on goals of other actors are not modeled. 


\subsection{Case Study Two: eSAP System}

The second example case is the eSAP system used as the case study system in [25, 26, 27]. The electronic Single Assessment Process (eSAP) system is an agent-based health and social care system for the effective care of older people. Taking into account a substantial part of the eSAP in the original case study, the following stakeholders are defined: The Older Person actor is the old person (patient) that wishes to receive appropriate health and social care. The Professional actor represents health and/or social care professionals involved in the care of the Older Person. The DoH actor represents the English Department of Health, which is responsible for the effective care of the Older Person. The Benefits Agency actor is an agency that helps the Older Person. Financially, and the R\&D Agency actor represents a research and development agency that is interested in obtaining medical information.

The approach proposed in [26, 27] integrates security concerns into the Tropos framework. Security constraints are modeled in a diagram called the actor diagram Security requirements and goals are expressed by "constraints", which are represented using a cloud placed between the dependencies, at the side of the actor who has to satisfy the constraint. This approach is useful, since it enables the designer to relate the security requirements to goals and functionalities.

The approach in [26, 27] introduces "security diagram”, which uses i* notation to model security requirements and potential threats. Fig. 11 gives the original security diagram of eSAP, which contains the security goals, threats, and safeguards. Threats are modeled explicitly in the "security diagram" using a pentagon. The impact of threats on softgoals such as privacy and availability is modeled using contribution links.

However, the model does not trace the threats to the source actors, and the relation between countermeasures and threats are not elaborated in the resulting models. The main lack of the security diagram in the original case study is that the resulting models do not include actors; therefore, it is not clear who has the goals, who threatens the goals and why, who employs the mechanisms, and what is the impact of the mechanisms on other goals of actors.

In [26, 27], once the stakeholders' goals and security constraints are identified, each actor is studied in more depth and alternative security solutions are examined considering the constraints imposed to the actor. Then, secure entities are introduced to help satisfying the imposed security constraints. However, it is not discussed why security requirements and constraint need to be distinguished by other softgoals.

Fig. 12 depicts one of the actor analysis models taken from [27]. The actor analysis model lacks considering the impact of security goals on attacks and threats. Therefore, alternative security solutions are modeled through means-end relations without the trade-offs that they impose on other goals and softgoals. 


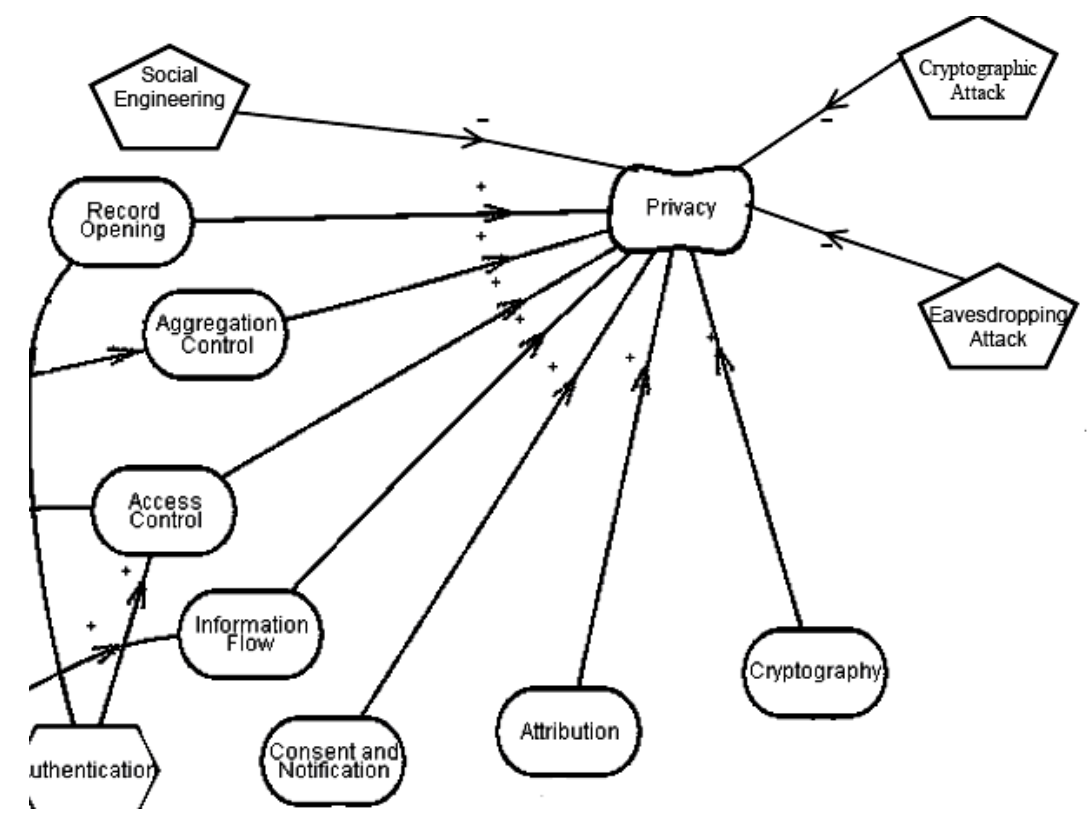

Fig. 11. A part of the "security diagram" for the eSAP system, original model in [27].

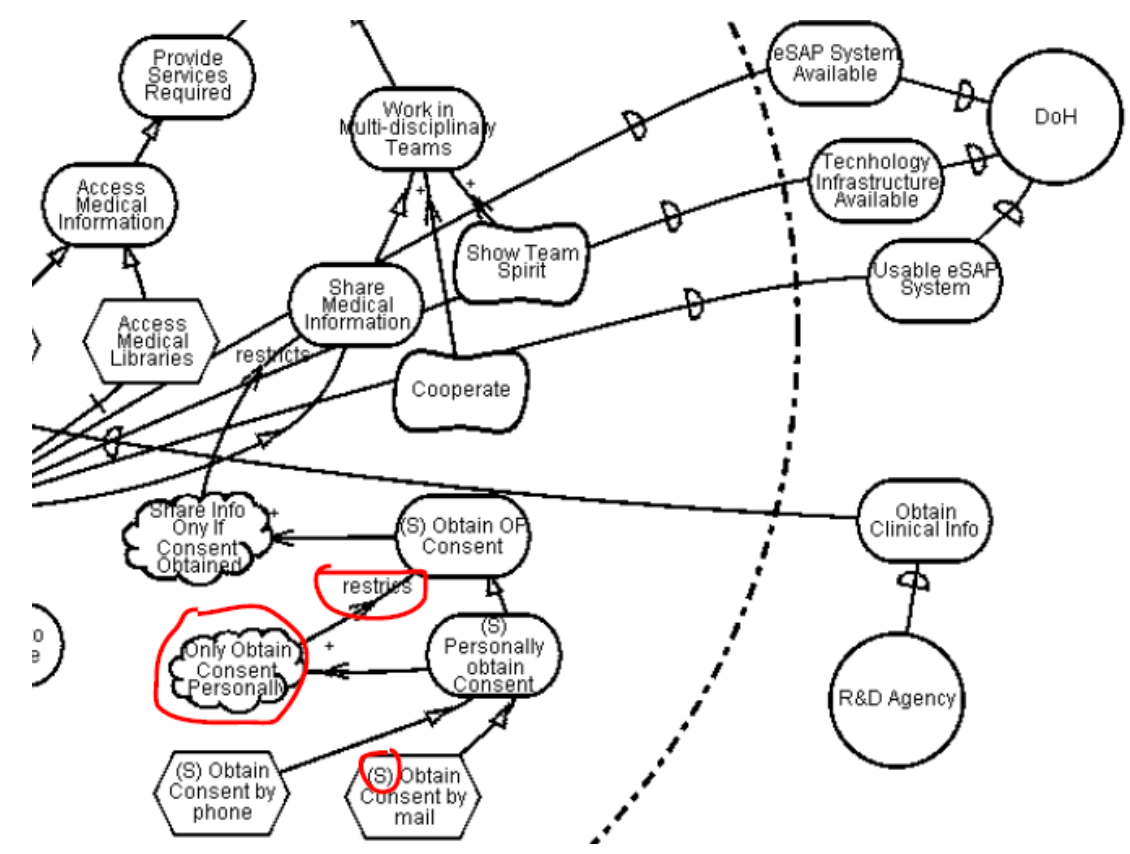

Fig. 12. Professional actor analysis model, from [27]. 
Fig. 13 illustrates the goal model of professional actor, developed using the proposed extensions to $i^{*}$. This model includes threats, security mechanisms and contributions of goals and attacks. This model includes the malicious tasks and goals and the corresponding impact on goals of professional actor. Fig. 13 illustrates a part of security trade-off analysis for eSAP system using the proposed approach. The steps of goal model evaluation are indicated by the tags.

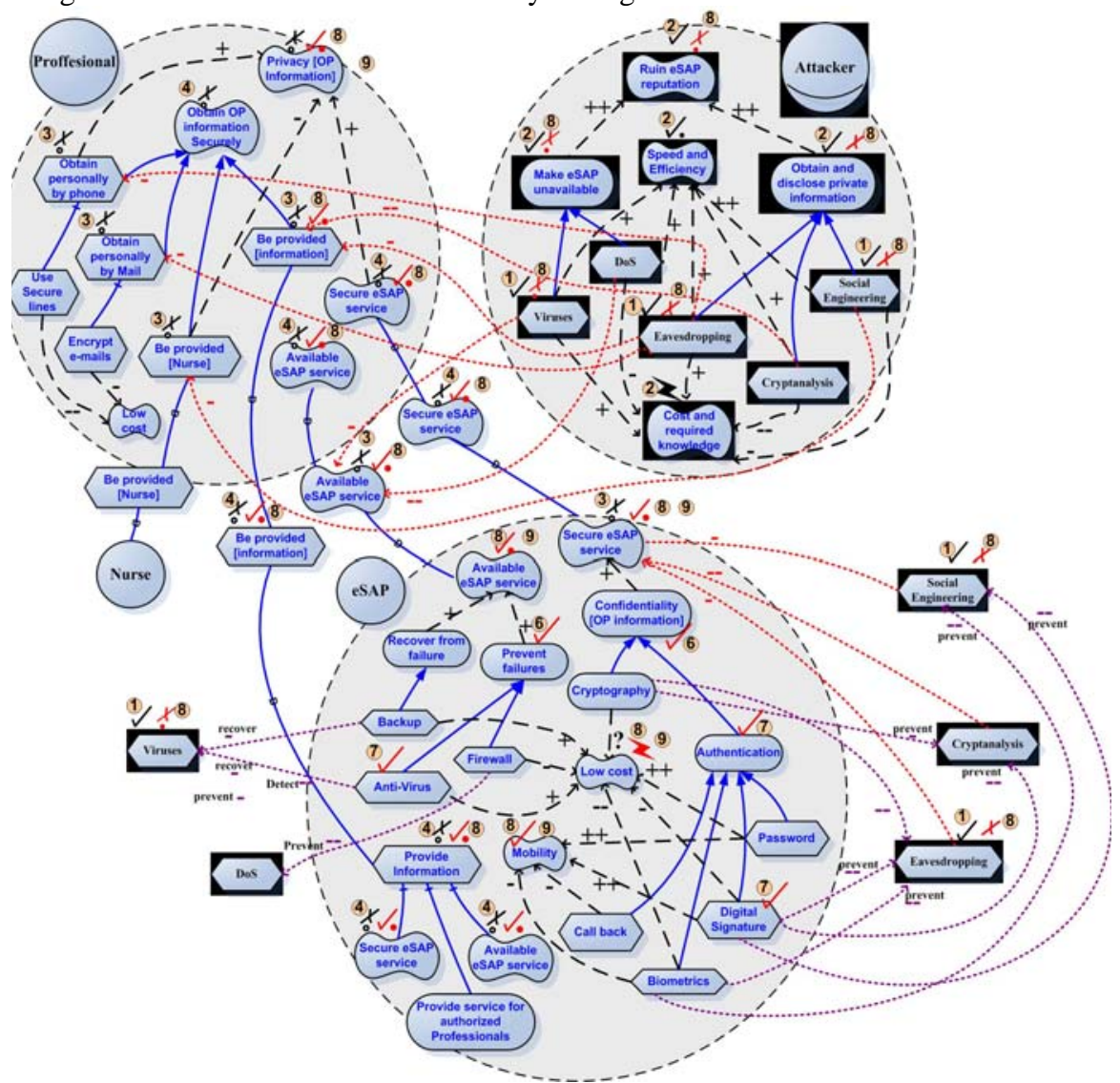

Fig. 13. Interaction of Attacker, professional and eSAP actors, and security goals and trade-offs, modeled using the proposed notation.

\subsection{Case Study Three: Course Registration System}

To our knowledge, the ATAM method provides is not applied in a security-specific case study. In this part, we apply the ATAM approach, using the notion of scenarios and tactics, to an example system. We use a simple course registration system to illustrate and compare the ATAM and our proposed approach. In this system, students 
take or drop courses by choosing them from a course catalogue, the system's admin edit course catalogues, and professors post final marks to the system. The privacy policies do not allow the system's admin to view academic history of students, but professors have access to the marks of their own courses. Academic history of students is personal information and should not be disclosed to the other students.

In what follows, the resulting artifacts and models developed by using the framework in [28] and our proposed approach are presented. First the models and artifacts which express the security requirements and design goals are given. Then the security solution models and trade-off analysis results are presented.

In [28], quality requirements are expressed as scenarios. Table 3 summarizes security and performance scenarios for the example course registration system. Scenarios provide the means to express requirements by specifying a circumstance and expected behavior of the system in that context. The circumstances are described by a stimulus and its source. To express malicious actors' behavior, threat can be expressed by the stimulus whose source is the attacker. However, this approach does not provide means to express the consequences of the attacks on other requirements.

Table 3. Scenarios for course registration case study

\begin{tabular}{|c|l|l|l|}
\hline Source & \multicolumn{1}{|c|}{ Scenario 1 } & \multicolumn{1}{|c|}{ Scenario 2 } & \multicolumn{1}{c|}{$\begin{array}{c}\text { Scenario 3 } \\
\text { (performance) }\end{array}$} \\
\hline Stimulus & $\begin{array}{l}\text { Tries to crack a } \\
\text { password }\end{array}$ & $\begin{array}{l}\text { Tries to sniff } \\
\text { transaction }\end{array}$ & Stochastic event \\
\hline Artifact & System data & Communicated data & Course Registration System \\
\hline Environment & Open/online & Open/online & Normal mode \\
\hline Response & $\begin{array}{l}\text { Keep the password } \\
\text { secret }\end{array}$ & $\begin{array}{l}\text { Protect confidential } \\
\text { data }\end{array}$ & $\begin{array}{l}\text { Process the requested } \\
\text { transaction }\end{array}$ \\
\hline $\begin{array}{l}\text { Response } \\
\text { Measure }\end{array}$ & $\begin{array}{l}\text { Password is } \\
\text { remained secret }\end{array}$ & $\begin{array}{l}\text { No confidential data } \\
\text { is revealed }\end{array}$ & $\begin{array}{l}\text { Latency: User waiting time } \\
\text { should be less than 1 minute }\end{array}$ \\
\hline
\end{tabular}

The approach in [28] suggests expressing security design solutions in terms of tactics. ATAM helps the designer evaluate each alternative tactic. The evaluator creates a "Quality Attribute Utility" tree where utility is the expression of the overall goodness of the system. A utility tree begins with utility as the root node. Quality requirements are placed as children of utility. Scenarios are placed at leaves of the tree. Fig. 14 depicts the utility tree for the example system. The difficulty to achieve and importance of each scenario is ranked by high/medium/low scheme. 


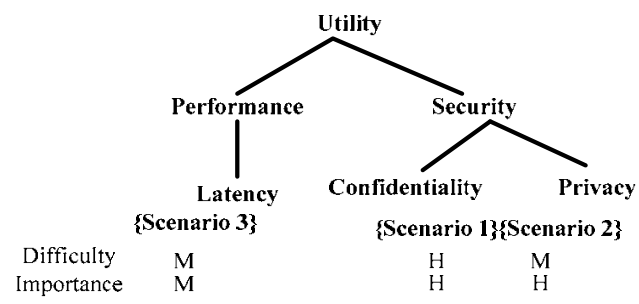

Fig. 14. ATAM utility tree for the quality requirements of Course Registration System

Based on the ATAM recommendations, scenarios that have at least one high priority of importance or difficulty should be analyzed in more detail to examine if the decided tactics satisfy the scenario. The result is an architectural approach analysis table for each scenario. Table 4 presents the architectural approach analysis table for the case study system.

Table 4. Architectural approach analysis for Course Registration case study compares alternative tactics

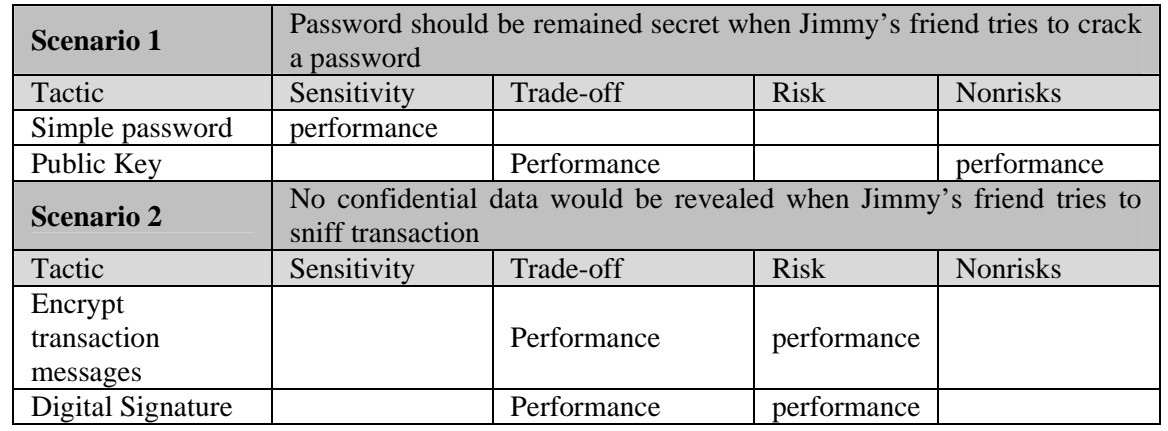

The utility tree does not model satisfaction degree of scenarios and contributions of quality requirements on each other. Besides, the utility tree approach does not specify which actor imposes the scenarios; therefore, the conflicts and trade-offs among goals of various stakeholders are not expressed explicitly. In this way, trade-offs among various quality scenarios are not expressed.

The "architectural approach analysis" table does not provide means to express the impacts of each tactics on stimuli (attacks). Trade-offs among quality scenarios and tactic in the architectural approach analysis table are expressed indirectly and implicitly, since trade-off and risk points, instead of referring to quality scenarios, point to affected quality properties. Moreover, the table does not capture the degree of sensitivity or trade-off between tactics and qualities. The analysis method does not provide any means to add qualitative or quantitative measures about the success or impacts of tactics and stimuli.

Fig. 15 depicts the malicious goal model affecting Jimmy's goals. This model includes threats, security design alternatives, and the result of trade-off analysis using 
security goal model evaluation. The selected alternatives and their impacts on threats are specified using the evaluation labels. The result of evaluation shows that Encrypt transaction messages and Public Key mechanisms result in losing performance. The implementation cost is hurt by Public Key mechanism and is improved by Encrypt transaction messages; therefore, there is a conflict on achieving implementation cost goal. On the other hand, both possible attacks (Sniff transactions and Crack Jimmy's password) would be controlled by these mechanisms, and performance goal is partially denied.

This model and the result of ATAM analysis in Table 4 result in similar conclusions. However, the goal model enables designers to express the trade-off rationale explicitly. Security goal models represent scenarios in terms of goals and softgoals, attack scenario in terms of malicious goals, and tactics in terms of security mechanisms. In the goal models, trade-offs among goals are expressed using contribution links. The results of goal model evaluation, which are assigned as satisfaction or denial labels to the goal graph elements, represent the trade-off analysis results graphically and explicitly.

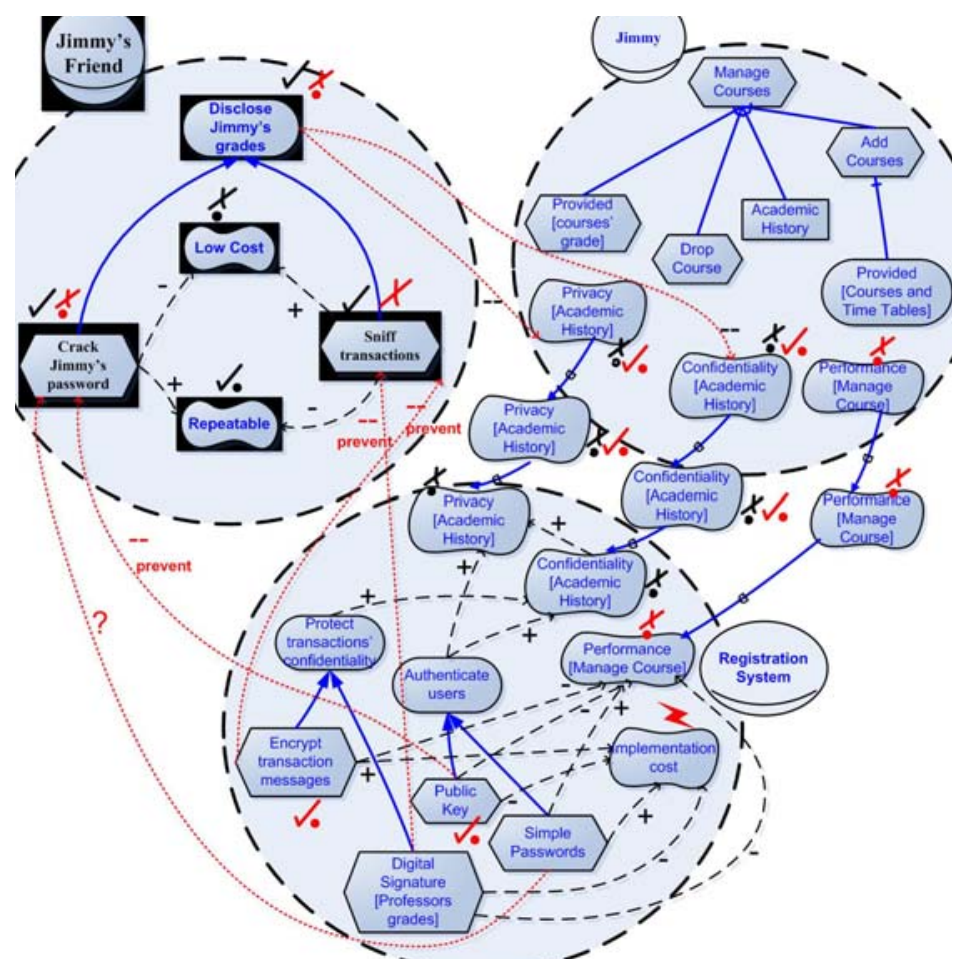

Fig. 15. Security goals and trade-offs, modeled using the proposed notation. The impact and trade-offs of each security alternative are modeled using the contribution links. 


\subsection{Summary of the Results}

The case studies and comparison of resulting models reveals strong and weak points of the previous works our proposed technique. The resulting models using the approach developed by Liu et al. [18] do not capture the goals and intentions behind malicious behavior. Therefore, the models do not specify how security alternatives protect the goals of actors against the threats. The models do not express which actor employs the security mechanisms, and the consequences of security mechanisms on goals of other actors are not modeled and analyzed. The current proposal resolves these expressiveness issues by separating malicious and non malicious actors and behavior.

In the models originally presented in [25, 26, 27], threats are not related to malicious actors who pose threats. This reduces the expressiveness and understandability of the models. Besides, the impacts of security mechanisms on threats are not expressed; therefore, the designer makes the trade-offs while the effectiveness of alternative solutions to protect the actors' goals against the threats is not considered.

The resulting artifacts of applying ATAM [28] for security trade-off analysis do not express security-specific aspects such as threats and vulnerabilities explicitly. Therefore, the artifacts do not capture the impact of threats on the quality goals of actors. In the ATAM approach, trade-offs are listed in tables for one scenario at a time. There are not explicit models of trade-offs across scenarios.

In the method proposed in this paper, threats are assigned to malicious actors, and the impacts of malicious behavior are expressed as contributions links from the malicious actors' boundaries to other actors' elements. Alternative solutions are selected considering potential goals and behavior of the attackers. In addition, tradeoffs that mechanisms impose are incorporated into the models by contributions of mechanisms and goals on softgoals.

\section{Discussions, Conclusions, and Future Work}

In this paper, we began by considering the criteria for a conceptual modeling technique that enables designers to model and analyze security trade-offs among competing goals of multiple actors to achieve a good enough security. We studied existing approaches to trade-off analysis and identified limitations of these approaches. Based on the evaluation criteria and limitation of previous works, we proposed extensions to the $i^{*}$ notation for modeling and analyzing security trade-offs of a multi-actor system. The proposed modeling notation is accompanied with a qualitative trade-off analysis procedure based on goal model evaluation methods. The procedure provides the designers with assessment of security mechanisms' impact on actors' goals and threats.

In the proposed notation, malicious and non-malicious elements are distinguished in the meta-model and the graphical representation. However, the definition of attack and security countermeasure is fundamentally a matter of perspective. A task or a 
goal counted as malicious can be perceived non-malicious from a different viewpoint. The sequences of actions for mounting an attack are similar to sequences of actions performed by non-malicious actors, and therefore, the line to differentiate malicious actions from non-malicious ones is arbitrary.

Other factors make establishing a definition for malicious behavior difficult. For instance, the concept of competition can be perceived comparable to the notion of attack, since competitions have similar impacts that attacks have, while the competitions are not malicious, illegal, or unauthorized. Since, it is a matter of perspective, distinguishing the malicious and non-malicious behavior does not affect the analysis. Malicious elements have the same semantics of ordinary intentional elements and they can be similar or identical to non-malicious elements.

On the other hand, as discussed by Sindre and Opdahl [40], graphical models become much clearer if the distinction between malicious and non-malicious elements is made explicit and the malicious actions are visually distinguished from the legitimate ones. In particular, Sindre and Opdahl show that the use of inverted elements strongly draws the attention to dependability aspects early on for those who discuss the models.

The proposed method in this paper suggests that analysts collect and model stakeholders and attackers goals and tasks for each project to analyze the trade-offs. Proposals such as SVDT [7] suggest using a single fixed set of pre-identified goals with their relationships and trade-offs expressed in a fixed BBN topology. The BBN design is unchanging and reused for every project. In such an approach, the trade-off model is a parametric (and possibly probabilistic) formula rather than a conceptual model. If the right topology that is applicable for all contexts is designed, it would save the analysts efforts to develop detailed goal models for every project.

Approaches such as SVDT, ATAM, and CBAM do not provide modeling techniques to express, extract, and resolve trade-offs among quality goals. Developing a specific goal model for every context and problem provides a deeper understanding for the analysts. Goals and requirements that are unique to a project can be dealt with specifically. Given the differences in these approaches, the usefulness of each approach for various contexts needs to be evaluated in practice.

In the current proposal, although the $i^{*}$ notation provides a proper basis for modeling and analyzing trade-offs, the models become complex and cumbersome when the goal models reach a certain size. Another limitation of the proposed approach is that a comprehensive source of knowledge of security mechanisms and corresponding contributions does not exist. In future work, we aim to conduct empirical studies of how security designers make trade-offs in practice, to adopt the proposed systematic trade-off analysis framework for integration into everyday design practice. We aim to build a security requirements and design knowledge base to gather and catalogue reusable knowledge about security trade-offs. Tool support for managing and applying security knowledge will also be studied. In this paper, the meta-model is mapped to the extended $i^{*}$ graphical representation. In future work, we aim to develop formal semantics for the meta-model in order to support automated reasoning. 
Acknowledgments. Financial support from Natural Science and Engineering Research Council of Canada and Bell University Labs is gratefully acknowledged.

\section{References}

1. Sasse, M. A., Computer Security: Anatomy of a Usability Disaster, and a Plan for Recovery, Workshop on Human-Computer Interaction and Security Systems, CHI 2003, Fort Lauderdale (2003)

2. DeWitt, A. J., Kuljis, J., Aligning Usability And Security-A Usability Study Of Polaris, In Proc. of the Symp. On Usable Privacy and Security (2006)

3. Sandhu, R., Good-Enough Security: Toward a Pragmatic Business-Driven Discipline," IEEE Internet Computing, Vol. 07, No. 1 (2003) 66-68

4. Houmb. S. H., Braber. F. D., Lund. M. S., Stølen. K., Towards a UML Profile for ModelBased Risk Assessment, In Proc. UML'2002 Satellite Workshop on Critical Systems Development with UML, (2002) 79-91

5. Butler, S. A., Security Attribute Evaluation Method: a Cost-Benefit Approach. In Proceedings of the International Conference on Software Engineering (ICSE'02), ACM Press (2002) 232-240

6. Alexander, I., Initial Industrial Experience of Misuse Cases in Trade-Off Analysis, In Proc. of the 10th Anniversary IEEE Joint Int. Conf. on Requirements Engineering (2002) 61-70

7. Houmb, S. H., Georg, G., Jürjens, J., France, R., An Integrated Security Verification and Security Solution Design Trade-off Analysis, In Integrating Security and Software Engineering: Advances and Future Visions, IDEA Group Publishing (2007) 190-219

8. Schneier, B., Attack Trees, 21-29. Dr. Dobb's Journal of Software Tools 24, 12 (1999) 21-29

9. Phillips, C. and Swiler, L. P. A graph-based system for network-vulnerability analysis, In Proc. of the 1998 Workshop on New Security Paradigm (1998) 71-79

10. Sindre, G., Opdahl, L., Eliciting security requirements by misuse cases, Proceedings of TOOLS Pacific, (2000) 120-131

11. McDermott, J., Fox, C., Using Abuse Case Models for Security Requirements Analysis, In Proc.15th. IEEE Annual Computer Security Applications Conf. (1999) 55-64

12. van Lamsweerde, A., Elaborating Security Requirements by Construction of Intentional Anti-models, In Proc. of ICSE’04 (2004) 148-15

13. Jürjens, J., Secure Systems Development with UML, Springer Academic Publishers, Germany (2004)

14. Lodderstedt. T., Basin. D., Doser. J., SecureUML: A UML-Based Modeling Language for Model-Driven Security, In Proc. of the 5th Int. Conf. on The Unified Modeling Language, Springer LNCS 2460 (2002) 426-441

15. Mayer, N., Rifaut, A., Dubois, E., Towards a Risk-Based Security Requirements Engineering Framework, 11th Int. Workshop on Requirements Engineering: Foundation for Software Quality (REFSQ'05) (2005)

16. Johnson, P., Lagerstrom, R., Norman, P., Simonsson, M., Extended Influence Diagrams for Enterprise Architecture Analysis, In: Enterprise Distributed Object Computing Conference, EDOC '06. 10th IEEE Int. (2006) 3-12

17. Liu, L., Yu, E., Mylopoulos, J., Analyzing Security Requirements as Relationships among Strategic Actors, In 2nd Symp. on Requirements Engineering for Information Security (SREIS) (2002)

18. Liu, L., Yu, E., Mylopoulos, J., Security and Privacy Requirements Analysis within a Social Setting. In IEEE Joint Int. Conf. on Requirements Engineering (2003) 151-161 
19. Giorgini, P., Massacci, F., Mylopoulos, J., Zannone, N., Modeling Security Requirements through Ownership, Permission and Delegation. In: 13th IEEE Int. Requirements Engineering Conf. (2005) 167-176

20. Haley, C. B., Laney, R. C., Moffett, J. D., Nuseibeh, B., The Effect of Trust Assumptions on the Elaboration of Security Requirements, In Proc. of the Requirements Engineering Conf. (RE'04) (2004) 102-111

21. Dardenne, A., van Lamsweerde, A., Fickas, S., Goal-Directed Requirements Acquisition, in The Science of Computer Programming 20 (1993) 3-50

22. Chung, L., Nixon, B. A., Yu, E., Mylopoulos, J., Non-Functional Requirements in Software Engineering, Kluwer Academic Publishing (2000)

23. Yu, E., Modeling Strategic Relationships for Process Reengineering, $\mathrm{PhD}$ thesis, Department of Computer Science, University of Toronto, Canada (1995)

24. Castro, J., Kolp, M., Mylopoulos, J., A requirements-driven development methodology, In Proc. of the 13th Int. Conf. on Advanced Information Systems Engineering, CAiSE'01 (2001) 108-123

25. Bresciani, P., Giorgini, P., Mouratidis, H., On Security Requirements Analysis for MultiAgent Systems, Proc. of 2nd Int. Workshop on Software Engineering for Large-Scale MultiAgent Systems (SELMAS) (2003) 35-48

26. Mouratidis, H., Giorgini, P., Manso, G., Philp, I, A Natural Extension of Tropos Methodology for Modelling Security, In Proc. of the Workshop on Agent-oriented methodologies, at OOPSLA (2002) 91-103

27. Mouratidis, H., Giorgini, P., Manso, Modelling Secure Multiagent Systems, In the 2nd Int. Conf. on Autonomous Agents and Multiagent Systems (2003) 859 - 866

28. Bass, L., Clements, P., Kazman, R., Software Architecture in Practice, Second Edition, Addison Wesley (2003)

29. Houmb, S. H., G. Georg, G., The Aspect-Oriented Risk-Driven Development (AORDD) Framework, In Proc. of the Int. Conf. on Software Development (2005) 81-91

30. Susi, A., Perini, A., Mylopoulos, J., The Tropos Metamodel and its Use, Informatica No. 29 (2005) 401-408

31. Grance, T., Stevens, M., Myers, M., Guide to Selecting Information Technology Security Products, Recommendations of the National Institute of Standards and Technology, NIST Special Publication 800-36 (2003)

32. US-CERT Vulnerability Notes Database, United States Computer Emergency Readiness Team, http://www.kb.cert.org/vuls

33. Anderson, R., Security Engineering: a guide to Building dependable Distributed systems, John Wiley and Sons (2001)

34. Pfleeger, C. P., Pfleeger,S. L., Security in Computing, Prentice Hall, 3dr edition (2002)

35. Yu, E., Towards Modelling and Reasoning Support for Early-Phase Requirements Engineering, Proc. of the 3rd IEEE Int. Symp. on Requirements Engineering (1997) 226-235

36. Haley, C. B., Moffett, J. D., Laney, R., Nuseibeh, B., A framework for security requirements engineering. In Software Engineering for Secure Systems Workshop (SESS'06) (2006) 35-42

37. Moffett, J. D., Haley, C. B., Nuseibeh, B., Core Security Requirements Artefacts, Department of Computing, The Open University, Milton Keynes UK, Technical Report 2004/23 (2004)

38. Horkoff J., Using i* Models for Evaluation, Masters Thesis, University of Toronto, Department of Computer Science (2006)

39. Szolovits, P., Doyle, J., Long, W. J., Guardian Angel: Patient-Centered Health Information Systems: MIT/LCS/TR-604, Available at: http://www.ga.org/ga.

40. Sindre, G., Opdahl, A. L, Capturing dependability threats in conceptual modeling, Conceptual Modelling in Information Systems Engineering (2007) 247-260 